\title{
Prevalência da Paracoccidioidomicose em pacientes diagnosticados no Hospital Araújo Jorge em Goiânia-Goiás, Brasil
}

\author{
Sara de Paula FERREIRA ${ }^{1 \#}$ \\ Rodrigo da Silva SANTOS ${ }^{2 \#}$ \\ Leonardo Barcelos de PAULA ${ }^{3}$ \\ Angela Adamski da Silva REIS ${ }^{4}$
}

\begin{abstract}
${ }^{1}$ Departamento de Biologia, Pontifícia Universidade Católica de Goiás (PUC-Goiás).
${ }^{2}$ Departamento de Genética - Departamento de Bioquímica e Imunologia, Faculdade de Medicina de Ribeirão Preto (FMRP), Universidade de São Paulo (USP).

${ }^{3}$ Departamento de Genética - Faculdade de Medicina de Ribeirão Preto (FMRP), Departamento de Química - Faculdade de Filosofia, Ciências e Letras de Ribeirão Preto (FFCLRP), Universidade de São Paulo (USP).

${ }^{4}$ Professora Doutora e Orientadora. Departamento de Bioquímica e Biologia Molecular, Instituto de Ciências Biológicas (ICB), Universidade Federal de Goiás (UFG).
\end{abstract}

E-mail: angeladamski@gmail.com (Reis, AAS)

(\# Autoria compartilhada)

RESUMO: O fungo Paracoccidioides brasiliensis é um patógeno humano com ampla distribuição na América Latina. $\mathrm{O}$ fungo causa a paracoccidioidomicose quando propágulos da fase miceliana atingem os pulmões do hospedeiro. $\mathrm{O}$ presente estudo analisou os aspectos clínico-patológicos de pacientes diagnosticados com PCM no Serviço de Anatomia Patológica (SAP) do Hospital Araújo Jorge em Goiânia-Goiás, no período de 2000 a 2006. Os casos analisados foram levantados a partir de livros de registro do SAP e os dados clínico-patológicos obtidos a partir dos respectivos laudos médicos. Foram diagnosticados 77 casos de PCM, dos quais 94,8\% eram pacientes do sexo masculino e 5,2\% do sexo feminino. A faixa etária de maior incidência identificada está entre os 40 e 60 anos de idade. Quanto à localização das lesões, os maiores percentuais de casos foram encontrados na cavidade oral (25,97\%), na laringe (12,98\%), em linfonodos $(10,38 \%)$, na língua $(7,79 \%)$, na pele $(6,49 \%)$ e no lábio $(5,19 \%)$. Em relação à distribuição anual, o maior número de casos (18), foi detectado no ano 2000. A PCM não é objeto de vigilância epidemiológica rotineiramente, e os casos diagnosticados no Hospital Araújo Jorge não são devidamente informados, por não dispor de instrumento de prevenção e por ser especializado preferencialmente no tratamento oncológico. No Brasil, tem-se registro de mais de 50 casos de PCM associados à AIDS o que coloca essa infecção como mais um indicador da síndrome. 100 anos após a sua descrição, a paracoccidioidomicose segue sendo uma enfermidade que requer considerações, interesse continuado e abordagem multidisciplinar.

Palavras-chave: Paracoccidioidomicose, Paracoccidioides brasiliensis, Hospital Araújo Jorge, Análise Epidemiológica.

\section{Prevalence of Paracoccidioidomycosis in patients diagnosed in Araujo Jorge Hospital, Goiânia-Goiás, Brazil}

\begin{abstract}
The fungus Paracoccidioides brasiliensis is a human pathogen widely distributed in Latin America. It is a deep mycosis and severe and may present associated with other diseases such as tuberculosis, AIDS and cancer. The present study analyzed the clinical and pathological aspects of patients diagnosed with PCM in the Service of Pathology Anatomy (SAP) of Araújo Jorge Hospital, Goiânia-Goiás, in the period 2000 to 2006. The cases analyzed were collected from the registration books of the SAP and the clinical and pathological data obtained from their medical records. We diagnosed 77 cases of PCM, of which $94.8 \%$ were male patients and $5.2 \%$ female. The age of highest incidence is identified between 40 and 60 years of age. As for the location of the lesions, the highest percentages of cases were found in the oral cavity $(25.97 \%)$, larynx (12.98\%), lymphnodes $(10.38 \%)$, tongue $(7.79 \%)$, the skin $(6.49 \%)$ and the lip (5.19\%). Regarding the distribution annual the largest number of cases (18) was detected in 2000. The PCM is not subject to routine surveillance, and cases diagnosed at the Hospital Araújo Jorge are not properly informed, for not having preventive tool and preferably be specialized in cancer treatment. In Brazil, has records of more than 50 cases of PCM associated with AIDS infection which puts this as another indicator of the syndrome. 100 years after its
\end{abstract}


description, paracoccidioidomycosis is still a disease that requires consideration, continuous interest and multidisciplinary approach.

Keywords: Paracoccidioidomycosis, Paracoccidioides brasiliensis, Hospital Araújo Jorge, Epidemiological Analysis.

\section{1- Introdução}

Paracoccidioides brasiliensis é um fungo dimórfico termo-regulado, patógeno humano e agente etiológico da paracoccidioidomicose (PCM), uma micose sistêmica geograficamente restrita a América Latina (Restrepo \& Tobón, 2005). O Brasil é responsável por $80 \%$ dos casos descritos na literatura, seguido por Colômbia e Venezuela (Coutinho et al., 2002). Os estados das regiões Sul, Sudeste e Centro-Oeste são, no Brasil, os locais onde a doença é mais frequentemente encontrada (Paniago et al., 2003). O fungo foi isolado pela primeira vez por Adolpho Lutz em 1908, tendo sido nesta ocasião se obtido o cultivo e se observado o aspecto dimórfico do fungo.

A infecção do hospedeiro humano geralmente ocorre pela inalação de propágulos do fungo. O fungo cresce como levedura nos tecidos infectados ou quando cultivado in vitro a $36^{\circ} \mathrm{C}$. A forma miceliana, considerada infectiva, é observada em condições saprobióticas no meio ambiente, ou quando cultivada em temperaturas inferiores a $28^{\circ} \mathrm{C}$ (San Blass et al., 2002). As células leveduriformes de $P$. brasiliensis são caracterizadas por apresentarem brotamentos múltiplos, formados pela evaginação da célula-mãe, onde uma célula central é circundada por várias células periféricas, conferindo um aspecto de roda de leme de navio. A forma miceliana pode ser identificada por filamentos septados com conídeos terminais ou intercalares (RestrepoMoreno, 2003).

O habitat exato de $P$. brasiliensis, ainda não está bem definido. No entanto, algumas hipóteses são propostas. Acredita-se que o fungo viva na natureza, em ambientes de água fresca, tais como rios ou córregos. Outra hipótese propõe que o fungo viva saprobiotícamente e de forma temporária no solo. Reforçando essa hipótese, o fungo foi isolado de duas espécies de tatus, o Dasypus novemcintus e o Cabassou centralis (Corredor et al., 2005).

O fungo $P$. brasiliensis pertence ao reino Fungi, filo Ascomycota, subdivisão Euascomycotina, classe Plectomyceto, subclasse Euascomycetidae, ordem Onygenales, família Onygenaceae, subfamília Onygenaceae Anamórficos, gênero Paracoccidioides, espécie Paracoccidioides brasiliensis (San-Blas et al., 2002). Recentemente, Matute et al., (2006) descreveram a existência de três diferentes espécies filogenéticas de $P$. brasiliensis: S1 (espécie 1), PS2 (espécie filogenética 2) e 168 
PS3 (espécie filogenética 3). A espécie filogenética PS3 está geograficamente restrita à Colômbia, enquanto S1 está distribuída no Brasil, Argentina, Paraguai, Peru e Venezuela. Alguns isolados da espécie filogenética PS2 foram encontrados no Brasil nos estados de São Paulo e Minas Gerais e ainda na Venezuela.

A primeira interação parasitahospedeiro ocorre nos alvéolos pulmonares; a partir dos pulmões, o fungo pode disseminarse por vias hematogênica ou linfática acometendo outros órgãos e sistemas como fígado, baço, ossos e sistema nervoso central (Valera et al., 2008).

A PCM atinge a pele, nódulos linfáticos e vários órgãos internos, incluindo os pulmões e o sistema nervoso central (Restrepo, 1985). O grau da patogênese varia

\section{2 - Material e Métodos}

O presente estudo analisou os aspectos clínico-patológicos de pacientes diagnosticados com PCM no Serviço de Anatomia Patológica (SAP) do Hospital Araújo Jorge em Goiânia-GO, no período de 2000 a 2006. Os casos analisados foram levantados a partir dos livros de registro do SAP e os dados clínico-patológicos obtidos a partir dos respectivos laudos. Os dados de acordo com as características do hospedeiro e da linhagem infectante. A resposta imune do hospedeiro contra o $P$. brasiliensis depende de fatores como idade, sexo, estado nutricional e herança genética. A PCM pode ser restrita ao trato respiratório ou pode disseminar-se através do organismo tornando-se letal (Franco, 1987).

A doença tem, em geral, evolução crônica, caráter recidivante e pode deixar sequelas anatômicas e funcionais. É grande a quantidade de doentes que necessitam de assistência médica de longo prazo nas regiões de maior endemicidade, tornando a moléstia, pela sua prevalência, um importante problema de saúde pública.

colhidos foram tabulados e analisados por meio de estatística descritiva.

Foram analisados todos os casos diagnosticados pelo SAP, cujos laudos faziam parte do arquivo do Hospital Araújo Jorge. Dentre os aspectos analisados, destacam-se a distribuição por sexo, faixa etária e local da lesão, presença ou não de neoplasia nos pacientes com paracoccidioidomicose e a distribuição anual dos casos. Foram 
detectados 77 pacientes com o diagnóstico de paracoccidioidomicose, os quais apresentavam sintomas clínicos físicos, que são as lesões comuns à patologia, como a principal forma de manifestação da doença.

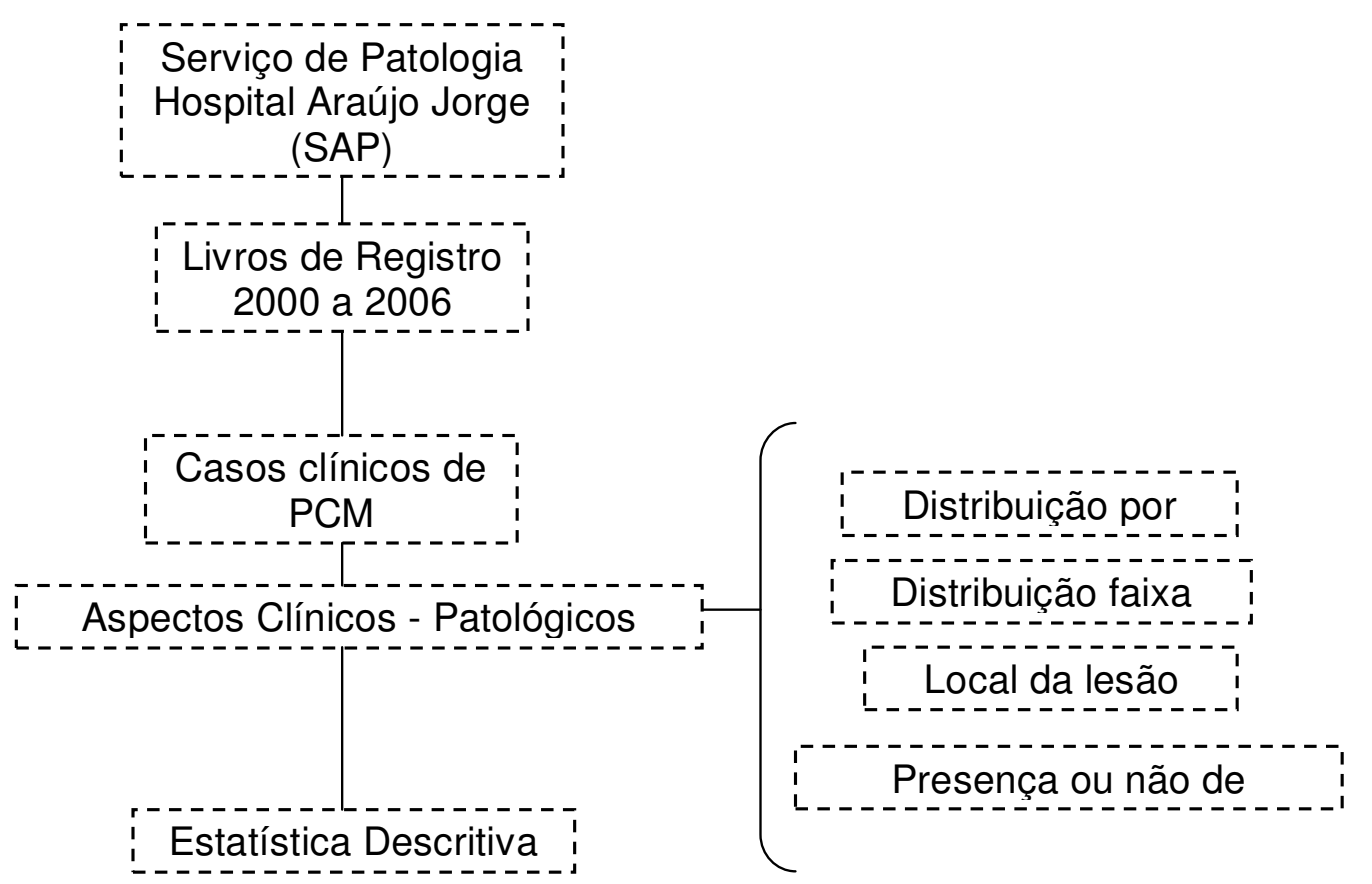

Figura 1: Fluxograma representativo das metas experimentais realizadas neste trabalho

\section{3- Resultados e Discussão}

No estudo de prevalência da PCM no Serviço de Anatomia Patológica do Hospital Araújo Jorge no período de 2000 a 2006 foi verificado 77 casos, sendo que na distribuição por sexo obteve-se uma proporção de 18:1, corroborando com os dados descritos na literatura, na qual o sexo masculino foi o mais acometido pela patologia quando comparado ao feminino, sendo 95\% (73/77) e 5\% (4/77), respectivamente. Campos et al., (1991) demonstraram dados semelhantes aos nossos. Mesquita et al., (1997) apresentaram um resultado acima do obtido pelo nosso estudo, no qual os autores relacionam tal resultado, com o fato à maior exposição do homem com o solo, e ser a mulher menos susceptível à doença, provavelmente pela proteção relacionada aos fatores hormonais e resposta fagocitária. Já na faixa etária pediátrica tanto a doença como a infecção incidem igualmente em ambos os sexos.

A faixa etária variou de 5 a 77 anos, sendo que a maior frequência situa-se na quarta e quinta décadas de vida (Tabela 1). A 
presença de 20/77 na quarta década e 30/77 na quinta década, totalizando 50/77 deles, confirmam os achados de alguns autores sobre a incidência da PCM (Mesquita et al., 1997 and Shikanai-Yasuda et al., 2006). Os estudos epidemiológicos realizados por Mesquita et al., (1997) apresentaram a presença de $45 / 58$ na faixa etária de 20 a 50 anos. Entretanto, nesse estudo obtiveram-se maiores casos na faixa etária de 40 a 60 anos e sendo todos do sexo masculino. Já para o sexo feminino a faixa etária em que prevaleceu a doença foi de 00 a 30 anos, sendo quatro pacientes femininos descritos (Tabela 1).

Tabela 1 - Distribuição dos casos de Paracoccidioidomicose dos pacientes diagnosticados no Hospital Araújo Jorge de acordo com a faixa etária.

\begin{tabular}{cccccccc}
\hline $\begin{array}{c}\text { Faixa } \\
\text { etária } \\
(\text { em }\end{array}$ & \multicolumn{3}{c|}{ Femininos } & \multicolumn{3}{c}{ Masculinos } & Total \\
\cline { 2 - 6 } anns) & $\mathrm{n}$ & $\begin{array}{c}\text { \% faixa } \\
\text { etária }\end{array}$ & $\begin{array}{c}\text { \% total } \\
\text { casos }\end{array}$ & $\mathrm{n}$ & $\begin{array}{c}\text { \% faixa } \\
\text { etária }\end{array}$ & $\begin{array}{c}\text { \% total } \\
\text { casos }\end{array}$ & \\
\hline $00-10$ & 1 & 25 & 1,3 & 3 & 75 & 3,9 & 4 \\
$11-20$ & 1 & 50 & 1,3 & 1 & 50 & 1,3 & 2 \\
$21-30$ & 2 & 66,6 & 2,6 & 1 & 33,3 & 1,3 & 3 \\
$31-40$ & 0 & 0 & 0 & 7 & 100 & 9,09 & 7 \\
$41-50$ & 0 & 0 & 0 & 20 & 100 & 25,9 & 20 \\
$51-60$ & 0 & 0 & 0 & 30 & 100 & 38,9 & 30 \\
$61-70$ & 0 & 0 & 0 & 6 & 100 & 7,7 & 6 \\
$71-80$ & 0 & 0 & 0 & 5 & 100 & 6,4 & 5 \\
\hline Total & 4 & 5,19 & 5,19 & 73 & 94,81 & 94,81 & 77 \\
\hline
\end{tabular}

O fungo $P$. brasiliensis parece crescer Quanto á distribuição das lesões provocadas

no solo como saprobiótico permanente, infectando o homem, principalmente por via inalatória. A progressão de infecção para doença depende de fatores relacionados tanto a virulência do patógeno, quanto a resposta imune do hospedeiro, podendo ocorrer depois de vários anos. Dessa forma, as manifestações clínicas da micose apresentam-se, na maioria das vezes, em pessoas acima dos 30 anos, apesar do contato inicial do hospedeiro com o fungo ter ocorrido durante as primeiras décadas de vida (Maluf et al., 2003). pela doença, os maiores percentuais de casos foram encontrados na cavidade oral $(25,97 \%)$, na laringe $(13,70 \%)$, linfonodos $(8,22 \%)$, na língua $(8,22 \%)$, na pele $(7 \%)$, na orofaringe $(6,85 \%)$ e no lábio $(4,11 \%)$. Múltiplos órgãos são acometidos e envolvidos durante o curso desta micose sistêmica (Tabela 2), sendo assim, os órgãos lesionados em decorrência da manifestação da PCM e que foram detectados neste estudo epidemiológico, encontram-se em concordância com a literatura (Mesquita et al., 1997; Bártholo et 
al., 2000; Telles-Filho 2001; Shikanai-Yasuda

et al., 2006).

Tabela 2 - Distribuição dos casos de Paracoccidioidomicose dos pacientes diagnosticados no Hospital Araújo Jorge de acordo com a localização das lesões.

\begin{tabular}{|c|c|c|c|c|}
\hline \multirow[t]{2}{*}{ Localização da lesão } & \multicolumn{2}{|c|}{ Sexo } & \multicolumn{2}{|c|}{ Total } \\
\hline & Feminino & Masculino & $\mathrm{N}^{\circ}$. & $\%$ \\
\hline Boca & & 1 & 1 & $1,37 \%$ \\
\hline Cápsula de abcesso cervical & & 1 & 1 & $1,37 \%$ \\
\hline Cavidade oral & & 20 & 20 & $25,97 \%$ \\
\hline Colón descendente & & 1 & 1 & $1,37 \%$ \\
\hline Corda vocal & & 1 & 1 & $1,37 \%$ \\
\hline Dorso do pé & & 1 & 1 & $1,37 \%$ \\
\hline Epiglote & & 2 & 2 & $2,74 \%$ \\
\hline Fígado & 1 & & 1 & $1,37 \%$ \\
\hline Fossa nasal & & 1 & 1 & $1,37 \%$ \\
\hline Gengiva & & 2 & 2 & $2,74 \%$ \\
\hline Intestino delgado - duodeno & 1 & & 1 & $1,37 \%$ \\
\hline Lábio & & 3 & 3 & $4,11 \%$ \\
\hline Laringe & & 10 & 10 & $13,70 \%$ \\
\hline Linfonodo axilar & & 2 & 2 & $2,74 \%$ \\
\hline Linfonodo cervical & 1 & 1 & 2 & $2,74 \%$ \\
\hline Linfonodo epitroclear & & 1 & 1 & $1,37 \%$ \\
\hline Linfonodo megalia & & 1 & 1 & $1,37 \%$ \\
\hline Língua & & 6 & 6 & $8,22 \%$ \\
\hline Loja amigdalina direita & & 1 & 1 & $1,37 \%$ \\
\hline Loja amigdalina esquerda & & 2 & 2 & $2,74 \%$ \\
\hline Orelha esquerda & & 1 & 1 & $1,37 \%$ \\
\hline Orofaringe & & 5 & 5 & $6,85 \%$ \\
\hline Palato & & 2 & 2 & $2,74 \%$ \\
\hline Palato duro & & 1 & 1 & $1,37 \%$ \\
\hline Palato mole & & 1 & 1 & $1,37 \%$ \\
\hline Pele - pálpebra - PS direita & & 1 & 1 & $1,37 \%$ \\
\hline Pele - região frontal & & 1 & 1 & $1,37 \%$ \\
\hline Pele-região superf. e frontal & & 1 & 1 & $1,37 \%$ \\
\hline Retroperitonela & 1 & & 1 & $1,37 \%$ \\
\hline Septo nasal & & 1 & 1 & $1,37 \%$ \\
\hline Supraglote & & 1 & 1 & $1,37 \%$ \\
\hline Tumor cervical SOE & & 1 & 1 & $1,37 \%$ \\
\hline Total & 4 & 73 & 77 & $100,00 \%$ \\
\hline
\end{tabular}

As lesões tegumentares ou cutâneomucosa são de grande importância no quadro clínico de PCM, sendo que as lesões da mucosa orofaríngea assumem um aspecto altamente sugestivo da doença (Bártholo et al., 2000). O envolvimento linfonodal é uma das manifestações clínicas mais comuns da PCM, demonstrando o tropismo eletivo do $P$. brasiliensis pelo sistema reticulo endotelial (Costa et al., 2005). A doença envolve 
primariamente os pulmões pela inalação, podendo posteriormente disseminar-se para vários órgãos e sistemas, originando lesões secundárias que ocorrem frequentemente nas mucosas, nos linfonodos, na pele e nas adrenais (Telles-Filho et al., 2001).

As lesões pulmonares são frequentes na PCM e quando ocorrem na forma crônica da doença, podem ser confundidas com a tuberculose, devido às semelhanças clínicas e radiográficas entre estas patologias (PalhetaNeto et al., 2003). Shikanai-Yasuda et al. (2006) destacam ainda que as manifestações pulmonares estão presentes em 90\% dos pacientes, e que, os pulmões, podem ser o único órgão afetado (unifocal) em até $25 \%$ dos casos. E geralmente, a doença envolve mais de um órgão simultaneamente (multifocal), sendo pulmões, mucosas e pele os locais mais acometidos pela infecção. Entretanto, neste estudo não obtivemos envolvimento pulmonar nos casos levantados.

Há registro na literatura de poucos casos de associação entre PCM e carcinomas, particularmente em localizações como pulmões, orofaringe e laringe. Mais raramente, neoplasias da série hematológica, como leucemia e linfoma, também podem ocorrer. A suspeita diagnóstica é fundamental para o estabelecimento precoce do diagnóstico e da terapêutica. A semelhança do que ocorre na tuberculose, a presença de carcinoma de pulmão em pacientes com PCM pulmonar pode ser mascarada por manifestações decorrentes da fibrose pulmonar consequente à micose. $\mathrm{O}$ mesmo pode ocorrer em lesões cicatriciais de acometimento de laringe e faringe, particularmente nos pacientes alcoolistas e tabagistas (Shikanai-Yasuda et al., 2006).

A partir da análise da distribuição anual dos casos de PCM diagnosticados no Serviço de Patologia do Hospital Araújo Jorge, verificou-se um maior número de registro de pacientes com a doença no ano 2000, com 18 casos, e no ano de 2006, com 12 casos. Entretanto, nos outros anos, 2001 a 2005, mantiveram-se uma quantidade de casos considerável: 10 em 2001, 11 em 2002, 11 em 2003, 8 em 2004 e 7 casos em 2005. A figura 2 demonstra esses dados distribuídos conforme o ano. 


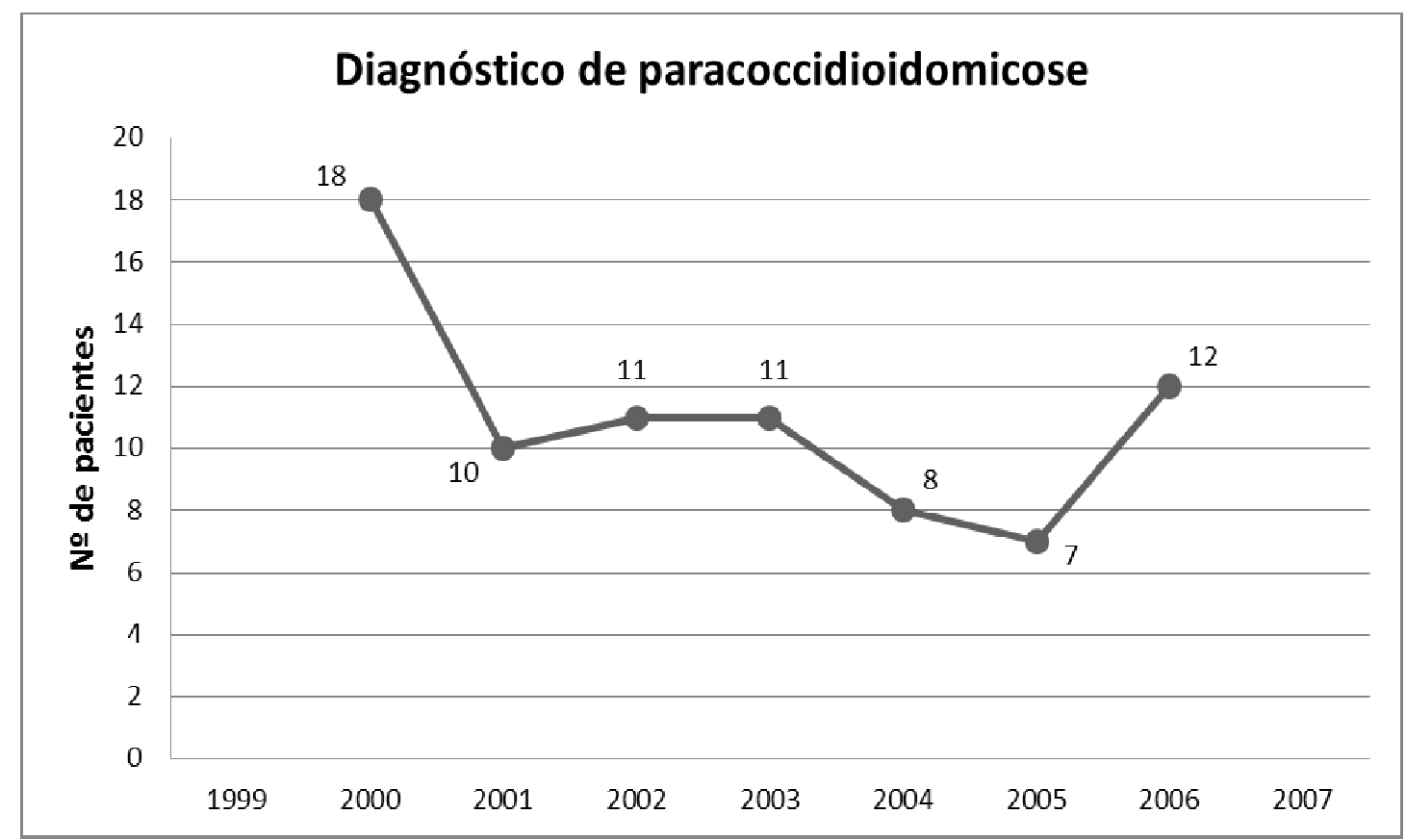

Figura 2 - Distribuição dos casos de Paracoccidioidomicose dos pacientes diagnosticados no Serviço de Patologia do Hospital Araújo Jorge, no período de 2000 a 2006.

Por não ser uma doença de notificação compulsória, torna-se difícil estabelecer a correta prevalência da paracoccidioidomicose no Brasil. Os dados existentes sobre sua distribuição geográfica e epidemiológica são fragmentários. Os cálculos de prevalência, incidência e morbidade da micose baseiam-se em relatos de inquéritos epidemiológicos e de séries de casos. Sua forma clínica mais comum reside nas lesões crônicas com alteração progressiva do estado geral, afetando com frequência os pulmões e a cavidade oral. Com base na experiência de serviços de referência no atendimento de pacientes com PCM, acredita-se que sua incidência em zonas endêmicas varie de 3 a 4 novos casos/milhão até 13 novos casos por
100 mil habitantes ao ano (Bisinelli et al., 2001 and Shikanai-Yasuda et al., 2006).

Os pacientes diagnosticados com PCM no Serviço de Patologia do Hospital Araújo Jorge não apresentavam doença de base como neoplasia, isso deve-se ao fato das lesões características da PCM serem associadas com lesões neoplásicas, assim justifica-se o diagnóstico destes pacientes em um hospital com área de atuação em oncologia e não em doenças tropicais. Desta forma, seria interessante e de grande importância à criação de equipes médicas e odontológicas multidisciplinares nas áreas endêmicas, para contribuir mais efetivamente com o diagnóstico e tratamento desta doença. 
A PCM é considerada como oitava causa de mortalidade por doença infecciosa, predominantemente crônica entre as doenças infecciosas e parasitárias e progressivamente incapacitante, (Shikanai-Yasuda et al., 2006) acomete o homem na fase mais produtiva de sua vida, gerando consequências sócioeconômicas não só à família, mas também ao

\section{4 - Conclusão}

O sexo e a idade são fatores determinantes na descrição do perfil epidemiológico da doença. Constitui grupo de risco o sexo masculino, com idade entre 40 e 60 anos de idade e associado à má higiene bucal, em função do número de lesões orais encontradas nos laudos. A PCM é uma doença que acomete preferencialmente homens, sendo de fácil disseminação e a manifestação sistêmica crônica da micose evolui para vários órgãos.

Nas lesões sistêmicas da forma crônica da micose, a cavidade oral, a laringe e o linfonodos foram os locais mais acometidos. A língua, a pele, a orofaringe e os lábios também foram regiões bastante comprometidas, entretanto não foi detectado paciente com acometimento pulmonar.
Estado (Mesquita et al., 1997). Até o momento, ainda não está estabelecido com precisão o hábitat do $P$. brasiliensis e nem um reservatório natural da doença entre os animais, o que dificulta a tentativa de interromper a cadeia de transmissão da doença.

Na distribuição anual, observou-se que a doença continua a infectar um número significativo de pessoas, mas não foi detectado nenhum caso com presença de neoplasia, uma vez que as lesões muitas vezes são associadas ao câncer e os pacientes são direcionados a procurar o Hospital Araújo Jorge de Goiânia para diagnóstico e tratamento.

A PCM é uma doença infecciosa grave e um importante problema de saúde pública, e por não ser uma doença de notificação compulsória, torna-se difícil estabelecer a correta prevalência e prevenção, necessitando de um trabalho mais eficiente para o Sistema de Informação do Ministério da Saúde. 


\section{Referências Bibliográficas}

Bártholo, RM et al. Um caso fácil: um diagnóstico rápido. Boletim de Pneumologia Sanitária, 8(2):28-37, 2000.

Bisinelli, JC and Ferreira, MLS. Doenças infecciosas: Paracoccidioidomicose (Blastomicose Sul-americana). In: TOMMASI, A. F. Diagnóstico em patologia bucal. $3^{\circ}$ ed. São Paulo: Pancast; 202-209, 2001.

Campos, EP et al. Paracoccidioidomicose: estudo radiológico e pulmonar de 58 casos. Revista do Instituto de Medicina Tropical de São Paulo, 33:267-276, 1991.

Corredor, GG et al. The naked-tailed armadillo Cabassous centralis (Miller 1899): a new host to Paracoccidioides brasiliensis. Molecular identification of the isolate.

Medical Mycology, 43:275-280, 2005.

Costa, MAB et al. Manifestação extrapulmonar da Paracoccidioidomicose. Radiologia Brasileira, 38(1):45-52, 2005.

Coutinho, ZF et al. Paracoccidioidomycosis mortality in Brazil (1980-1985). Caderno de. Saúde. Pública, 18:1441-1454, 2002.

Franco, M. Host-parasite relationship in paracoccidiodomycosis. Journal of Medical and Veterinary Mycology, 25:5-18, 1987.

Maluf, MLF et al. Prevalência de paracoccidioidomicose: infecção determinada através de teste sorológico em doadores de sangue na região noroeste do Paraná, Brasil. Revista da Sociedade Brasileira de Medicina Tropical, 36:11-16, 2003.

Matute, DR et al. Cryptic speciation and recombination in the fungus Paracoccidioides brasiliensis as revealed by gene genealogies.

Molecular Biology and Evolution, 23:65-73, 2006.
Mesquita, FCL et al. Paracaccidioidomicose. In: Leão RNQ, coordenador. Doenças infecciosas e parasitárias. Enfoque amazônico. Belém: Editora Cejup; 767-81, 1997.

Palheta-Neto, FX et al. Estudo de 26 casos de Paracoccidioidomicose avaliados no Serviço de Otorrinolaringologia da Fundação Oswaldo Cruz. Revista Brasileira de Otorrinolaringologia, 69(5):622-627, 2003.

Paniago, AM et al. Paracoccidioidomicose: estudo clínico e epidemiológico de 422 casos observados no Estado de Mato Grosso do Sul. Revista da Sociedade. Brasileira de Medicina Tropical, 36(4):455-459, 2003.

Restrepo A. The ecology of Paracoccidioides brasiliensis: a puzzle still unsolved.

Sabouraudia, 23(5):323-334, 1985.

Restrepo, A \& Tobón, A. Paracoccidioides brasiliensis. In: Mandell, GL; Bennett, JE; Dollin, R (Ed). Principles and Practice of Infectious Diseases, Philadelphia, 30623068, 2005.

Restrepo-Moreno,A. Paracoccidioidomycosis. In: Pappas, WE; Sobel, PG (ed.), Clinical Mycology. Oxford University Press, New York, 328-345, 2003.

San-Blas, G et al. Paracoccidioides brasiliensis and paracoccidioidomycosis: Molecular approaches to morphogenesis, diagnosis, epidemiology, taxonomy and genetics. Medical Mycology, 40:225-242, 2002.

Shikanai-Yasuda, MA et al. Guideliness in paracoccidioidomycosis. Revista da Sociedade Brasileira de Medicina Tropical, 39(3):297-230, 2006. 
Teles-Filho, FQ. Boletim Epidemiológico da Secretaria de Estado de Saúde do Paraná,

Curitiba, 2001.

Valera, ET, et al. Fungal infection by

Paracoccidioides brasiliensis mimicking bone

tumor. Pediatric Blood \& Cancer, 50:1284-

1286, 2008. 